\title{
Bilateral Augmentation of Dopaminergic and Serotonergic Activity in the Striatum and Nucleus Accumbens Induced by Conditioned Circling
}

\author{
C. Szostak, A. Jakubovic, A. G. Phillips, and H. C. Fibiger \\ Division of Neurological Sciences, Department of Psychiatry and Department of Psychology, \\ University of British Columbia, Vancouver, British Columbia V6T 1W5, Canada
}

The involvement of dopaminergic (DA) and serotonergic (5-HT) systems in circling was assessed by determining the neurochemical correlates of circling induced and maintained by two different schedules of water reinforcement. The conditioned circling paradigm was employed in an attempt to replicate reports that levels of DA and 3,4-dihydroxyphenylacetic acid (DOPAC) were increased in the striatum and nucleus accumbens septi (NAS) contralateral to the direction of circling. Rats trained to circle using a continuous schedule of reinforcement did not exhibit any changes in concentrations of DA, DOPAC, or homovanillic acid (HVA). Bilateral increases in 5-HT concentrations were observed in the striatum. Use of an intermittent schedule of reinforcement (FR-2) produced higher rates of circling. In rats maintained on the FR-2 schedule, no changes in DA or its metabolites were observed in the striatum. The ratio of HVA to DA was, however, increased bilaterally, suggesting a bilateral augmentation of DA utilization. Concentrations of DA were lower in the NAS contralateral to direction of turning. While NAS levels of HVA were elevated bilaterally when compared to noncircling controls, HVA was lower in the NAS contralateral to the direction of circling. DA utilization, as estimated by HVA: DA ratios, was increased bilaterally in the NAS. None of the measures of DA activity within the olfactory tubercle (OT) were influenced by circling. Turnover of 5-HT, as estimated by the ratio of 5-HT to 5-hydroxyindoleacetic acid (5-HIAA), was increased bilaterally in the striatum, NAS, and OT. Together, the present results indicate that $\mathrm{DA}$ function can be influenced by high rates of circling. However, contrary to previous reports, circling does not produce an imbalance in the activity of the nigrostriatal and mesolimbic DA systems. Instead, the activity of both systems appears to be enhanced bilaterally as a result of circling. In addition, ascending 5-HT projections appear to be involved in the performance of the circling response.

Identification of the neurochemical substrates of specific aspects of behavior has been sought predominantly through the use of pharmacological manipulations and selective lesion techniques. Evidence for the critical involvement of dopamine (DA) in movement is provided by the finding that unilateral lesions of the nigrostriatal DA projection by localized injection of the neurotoxin 6-hydroxydopamine (6-OHDA) elicits circling toward the lesioned side (Ungerstedt, 1971a). In addition, following the remission of spontaneous circling, rotation can be rein-

Received Aug. 26, 1985; revised Dec. 10, 1985; accepted Dec. 26, 1985.

This work was supported by MRC Program Grant 23, C.S. is a NSERC Postgraduate Scholar. The excellent technical assistance of D. Fu is gratefully acknowledged.

Correspondence should be addressed to C. Szostak, Division of Neurological Sciences, Department of Psychiatry, University of British Columbia, 2255 Wesbrook Mall, Vancouver, B. C., V6T IW5, Canada.

Copyright (C) 1986 Society for Neuroscience $0270-6474 / 86 / 072037-08 \$ 02.00 / 0$ stated by the systemic administration of DA agonists such as amphetamine and apomorphine. Moreover, the direction of druginduced circling depends on the type of agonist employed. That is, indirect agonists that influence release and reuptake of DA, such as amphetamine, produce circling toward the lesioned side (i.e., ipsilateral rotation), while directly acting postsynaptic receptor agonists such as apomorphine induce turning in the opposite direction (i.e., contralateral rotation) (Ungerstcdt, 1971a, b). On the basis of these results it has been concluded that animals typically circle away from the dominant side of DA activity.

Further evidence of dopaminergic involvement in circling is derived from reports that unilatcral microinjections of DA into the dorsal striatum produce contralateral turning (Joyce and van Hartesveldt, 1984; Joyce et al., 1982; Wolfson and Brown, 1983). Moreover, inhibition of DA synthesis or blockade of DA receptors results in an attenuation of drug-induced circling (Gershanik et al., 1983; Kelly and Moore, 1977). While a strong case has been made for an involvement of DA in circling, other systems have also been implicated. For example, drugs that act on serotonergic, cholinergic, noradrenergic, and GABAergic systems have been found to either evoke or modulate drug-induced rotation, indicating that DA is not uniquely involved (see Pycock, 1980, for review).

Since pharmacological treatments produce nonspecific behavioral and neurochemical effects, Yamamoto and Freed (1982, 1984) recently attempted to identify the neurochemical correlates of circling in undrugged and unlesioned animals. These authors reported that the performance of a conditioned circling response results in increased levels of DA and 3,4-dihydroxyphenylacetic acid (DOPAC) in the striatum and nucleus accumbens contralateral to the direction in which the rats turned. These results are consistent with the suggestion that circling involves an asymmetry in the activity of the nigrostriatal projection, as well as enhanced activity within the mesolimbic DA system (Kelly and Moore, 1977; Pycock and Marsden, 1978).

The findings of Yamamoto and Freed $(1982,1984)$ are also important, as they indicate that localized and specific neurochemical alterations are associated with the performance of a specific behavioral response. While enhanced synthesis and turnover of DA has been associated with lever-pressing (Heffner and Seiden, 1981; Heffner et al., 1981), changes in the concentrations of DA and its metabolites are not apparent in animals tested in a drug-free state (Heffner et al., 1984). Given that lateralized changes in DA and DOPAC levels can be reliably induced by the conditioned circling paradigm (Yamamoto and Freed, 1982, 1984), this procedure should provide a simple means for investigating further the neural substrates of movement.

The present series of experiments further examined the role of DA in conditioned circling. In the first experiment, rats were 
trained to circle using a continuous schedule of reinforcement in an attempt to replicate the basic findings of Yamamoto and Freed (1982), i.e., that rats trained to circle exhibit increased levels of DA and DOPAC in the striatum and nucleus accumbens contralateral to their direction of turning. A second experiment was conducted to assess the extent to which rate of circling would influence the neurochemical consequences of circling.

\section{Materials and Methods}

\section{Experiment 1}

It has been reported that rats trained to circle according to a continuous schedule of reinforcement (CRF) exhibit increased levels of DA and DOPAC in the striatum and nucleus accumbens contralateral to the direction in which they turn (Yamamoto and Freed, 1982). The first experiment was conducted to determine the reliability of this phenomenon.

\section{Subjects and apparatus}

Naive, male Sprague-Dawley rats (Charles River Laboratories), weighing 250-350 gm, served as subjects. Rats were individually housed in a temperature-controlled room with an automatic $12 \mathrm{hr}$ light/dark cycle (lights on: 0800-2000). Food and water were available ad libitum in the home cage. Animals were acclimatized to the laboratory for I week prior to commencement of the experiment.

All behavioral testing was conducted in a plastic cylindrical container (diameter, $26 \mathrm{~cm}$; height, $30 \mathrm{~cm}$ ). The container was equipped with a manually operated, motor-driven liquid dispenser (Skinner Electric Valve Division) calibrated to deliver $0.07-0.09 \mathrm{ml}$ of water per reinforcement. The water was delivered into a trough located at the perimeter of the apparatus $4 \mathrm{~cm}$ from the floor.

\section{Procedure}

Eight rats were water-deprived for $23 \mathrm{hr}$ prior to commencement of training. Subjects were trained to turn $360^{\circ}$ for water, using the method of successive approximations. The direction of turning was assigned randomly, such that half the rats were required to circle to the left and the other half to the right, as described by Yamamoto and Freed (1982). Upon acquisition of the appropriate response, each complete circle emitted was reinforced. Animals received eight to nine daily training sessions of $20 \mathrm{~min}$ duration. During the experimental regimen, food continued to be available ad libitum in the home cage. However, access to water was limited to that received in the test apparatus $(8-12 \mathrm{ml} / \mathrm{d})$. The imposed schedule of water deprivation resulted in animals maintaining $80-85 \%$ of their free-feeding body weights. On the day following the last training session (day 9-10), animals were tested for circling behavior for $20 \mathrm{~min}$. The experimental contingencies used during training remained in effect. Immediately after the session, rats were decapitated and their brains rapidly removed, mounted on a microtome, and frozen. Slices containing the left and right striata and nuclei accumbens septi (NAS) were placed on ice. These structures were then dissected from the slices and prepared for analysis of DA, DOPAC, homovanillic acid (HVA), 5-HT, and 5-hydroxyindoleacetic acid (5-HIAA) by high-pressure liquid chromotography with electrochemical detection (HPLC-ED), as described by Jakubovic et al. (in press).

Eight additional rats served as control subjects. These rats were placed on a $23.5 \mathrm{hr}$ water-deprivation schedule and handled daily for the duration of the experiment. Control subjects did not receive any behavioral training or experience in the test apparatus. On the test day, control subjects were decapitated after having access to water in their home cage for $20 \mathrm{~min}$. The brains of the control subjects were processed as described above.

\section{Experiment 2}

In this experiment, rats were trained to circle for water reinforcement as in the first experiment, except that an intermittent schedule of reinforcement was implemented, resulting in much higher rates of circling.

\section{Subjects and apparatus}

Naive, male Sprague-Dawley rats, as described in Experiment 1, served as subjects. The apparatus used for behavioral testing was as described previously.

\section{Procedure}

Five rats were randomly assigned to be trained to circle either to the left or to the right. Subjects were water-deprived for $24 \mathrm{hr}$ before commencement of training. Food was available ad libitum in the home cage. Each rat received five daily $20 \mathrm{~min}$ training sessions, wherein each complete circle performed was reinforced. On the sixth day of training, the first 10 rotations were reinforced according to a CRF schedule. Thereafter, the schedule of reinforcement was switched to a fixed ratio of 2 (FR-2), whereby every second rotation was reinforced. Training continued until every subject was consistently making a minimum of 300 rotations within a 20 min session (i.e., either 15 or 21 sessions). Training was interrupted for $5 \mathrm{~d}$ after the fifth FR-2 session, as the imposed schedule of water deprivation resulted in too severe a degree of weight loss. During this $5 \mathrm{~d}$ period, animals were placed on ad libitum food and water.

Animals were decapitated immediately following completion of the last session (day 15 or 21 of FR-2 training). The brains were rapidly removed and frozen. Left and right striata, NAS, and olfactory tubercle (OT) were dissected from the appropriate tissue slices, weighed, and prepared for analysis by HPLC-ED as in Experiment 1 .

Three additional rats served as control subjects as described in Experiment 1 . For purposes of analyzing the neurochemical data, the left and right tissue samples obtained from control subjects were randomly designated ipsilateral and contralateral.

\section{Results}

\section{Experiment 1}

All rats acquired the task and, by the fifth session, were performing at least 100 complete rotations in a 20 min session. Once they had acquired the correct response, circling in the inappropriate direction was rarely observed. Mean rotations per minute obtained during the test session are presented in Figure 1. An analysis of variance (ANOVA) with one within-subject factor (ten 2 min blocks) was conducted on the behavioral data obtained during the test session. Degrees of freedom appropriate to the Geisser-Greenhouse Conservative $F$ test were used for this analysis, and for all subsequent analyses involving withinsubject measures. Rate of circling decreased progressively during the session: $F(1,7)=19.07, p<0.05$. While the maximal rates of responding obtained in the present investigation are similar to those reported by Yamamoto and Freed (1982), animals in the present study exhibited a greater decline in responding within a session.

To determine whether reinforced circling selectively influenced the activity of DA, 5-HT, and their metabolites in the striatum and NAS contralateral to the direction in which they circled, ANOVAs with one between-subject factor (Group) and one within-subject factor (Ipsilateral vs Contralateral structure) were conducted for each compound measured by IIPLC-ED. Since there is evidence of neurochemical asymmetries in naive animals (Jerussi and Taylor, 1982; Rosen et al., 1984), the left and right tissue samples obtained from control subjects were randomly designated as ipsilateral and contralateral, such that half of the "ipsilateral" samples wcrc obtaincd from the right hemisphere and half from the left. One experimental subject was omitted from the NAS analyses because of an error made during the preparation of the contralateral NAS sample. In addition, NAS levels of 5-HT and 5-HIAA could not be determined for onc control subject. ANOVAs were also conducted on the ratios of a given metabolite to its parent transmitter substance, as it has been suggested that such ratios may be a more sensitive indicator of amine utilization (e.g., Lavielle et al., 1978).

Circling was not found to influence levels of DA, DOPAC, or HVA (see Table 1), nor did it alter the ratios of DOPAC or HVA to DA in either the striatum or the NAS. Experimental subjects were, however, found to have bilaterally elevated levels of 5-HT in the striatum, relative to control subjects: $F(1,14)=$ 
$4.866, p<0.05$ (see Table 2). The significance of the bilateral elevation of striatal 5-HT is not known. The ratio of 5-HT to 5-HIAA was not changed by circling, suggesting that 5-HT utilization was not modified during this behavior.

These data fail to confirm the reports of Yamamoto and Freed $(1982,1984)$ that conditioned circling produces lateralized increases in the concentrations of DA and DOPAC in the striatum and NAS. One possible explanation for this discrepancy is that the terminal rate of responding in the present study was lower than that obtained by Yamamoto and Freed. While the maximal rates of circling were similar, animals in the present study displayed a substantial reduction in turning behavior within the 20 min session, such that for the last $6 \mathrm{~min}$ of the session the rats performed an average of two to four circles a minute. For this reason, a second experiment was undertaken to assess whether lateralized neurochemical changes would be obtained in subjects exhibiting much higher rates of circling.

\section{Results}

\section{Experiment 2}

Behavior

Relative to the rates obtained when a CRF schedule was used, intermittent reinforcement of circling resulted in a marked increase in rates of responding throughout the session (Fig. 1). As in the first experiment, response rates were found to decline within a session: $F(1,4)=8.713, p<0.05$. The mean minimal response rate observed in the test session was slightly greater than the mean maximal rates (10-12 circles/min) reported by Yamamoto and Freed (1982). If response rates are critical in determining the neurochemical correlates of conditioned circling, then lateralized changes should be readily apparent in the present investigation.

\section{Neurochemistry}

Striatum. Analyses conducted on striatal concentrations of DA, DOPAC, and HVA failed to reveal any statistically significant effects (see Table 3). While DOPAC:DA ratios did not differ between groups, analysis of the HVA:DA ratios indicated that the conversion of DA to HVA was augmented bilaterally in rats trained to circle for water reinforcement: $F(1,5)=7.264, p<$ 0.05 (see Fig. 2). This finding suggests that high rates of circling, induced and maintained by water reinforcement, can result in an enhancement of striatal dopaminergic activity. However, this neurochemical effect is not restricted to the contralateral striatum, as suggested by Yamamoto and Freed $(1982,1984)$.

Striatal concentrations of 5-HT were not influenced by high

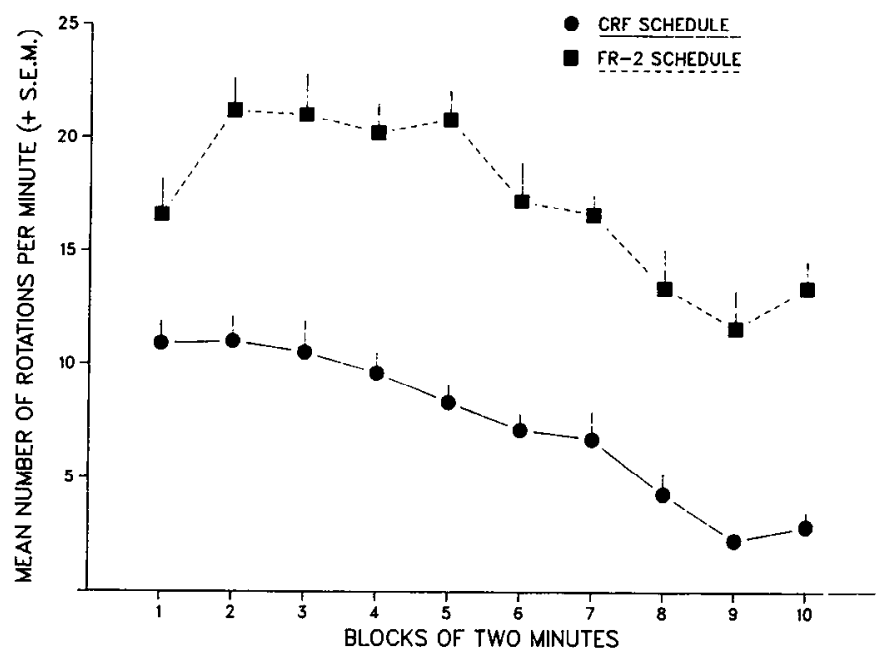

Figure 1. Number of rotations emitted by rats during test sessions. Values represent means (+SEM) and are presented in blocks of 2 min. Solid circles, Test data of rats trained to circle using a continuous schedule of reinforcement (Experiment 1); solid squares, mean response rates of rats trained to circle using an FR-2 schedule (Experiment 2).

rates of circling as they were in Experiment 1 (Table 4). Rather, a trend in the opposite direction was observed: $F(1,5)=4.995$, $0.05<p<0.10$. While 5-HIAA levels were not affected, the turnover of $5-\mathrm{HT}$, as estimated by 5 -HIAA : 5 -HT ratios, was enhanced bilaterally in rats trained to circle: $F(1,5)=22.42, p<$ 0.01 (Fig. 2).

Nucleus accumbens. Analysis of DA concentrations within the NAS yielded a statistically significant interaction: $F(1,6)=$ $6.402, p<0.05$ (Table 3). Tukey's pairwise comparisons $(\alpha=$ 0.05 ) indicated that the interaction reflects an asymmetry in DA concentrations in animals trained to circle, whereas ipsi- and contralateral levels of DA did not differ in control subjects. The direction of the asymmetry (i.e., a decrease in contralateral NAS as opposed to an increase in ipsilateral NAS) cannot be statistically determined, since neither of the experimental values differed from the appropriate control values. Inspection of the data does, however, indicate that levels tend to be lower in the contralateral NAS, relative to both the ipsilateral NAS and control values. The overall analysis conducted on DOPAC concentrations in the NAS did not yield statistically significant differences. However, analysis of HVA levels yielded a significant interac-

Table 1. Concentrations of DA, DOPAC, and HVA in the striatum and NAS of circling and noncircling control rats

\begin{tabular}{|c|c|c|c|c|c|c|}
\hline & \multicolumn{2}{|c|}{ Dopamine } & \multicolumn{2}{|c|}{ DOPAC } & \multicolumn{2}{|l|}{ HVA } \\
\hline & Ipsi & Contra & Ipsi & Contra & Ipsi & Contra \\
\hline \multicolumn{7}{|l|}{ Striatum } \\
\hline \multirow[t]{2}{*}{ Controls $(n=8)$} & 124.38 & 124.00 & 21.73 & 21.71 & 9.21 & 9.27 \\
\hline & 6.29 & 5.41 & 0.93 & 0.96 & 0.41 & 0.44 \\
\hline \multirow[t]{2}{*}{ Circlers $(n=8)$} & 121.75 & 120.63 & 22.71 & 22.40 & 10.01 & 9.85 \\
\hline & 1.96 & 3.08 & 1.17 & 1.23 & 0.43 & 0.39 \\
\hline \multicolumn{7}{|l|}{ NAS } \\
\hline \multirow[t]{2}{*}{ Controls $(n=7)$} & 85.85 & 90.50 & 22.41 & 23.04 & 7.54 & 7.77 \\
\hline & 3.89 & 6.43 & 0.76 & 1.37 & 0.48 & 0.42 \\
\hline \multirow[t]{2}{*}{ Circlers $(n=8)$} & 86.11 & 84.06 & 23.86 & 23.20 & 8.33 & 8.40 \\
\hline & 4.67 & 3.42 & 0.83 & 0.40 & 0.33 & 0.22 \\
\hline
\end{tabular}


Table 2. Concentrations of 5-HT and 5-HIAA in the striatum and NAS of circling and noncircling control rats

\begin{tabular}{llllll} 
& 5-HT & & & 5-HIAA \\
& Ipsi & Contra & & Ipsi & Contra \\
\hline Striatum & & & & \\
Controls $(n=8)$ & 1.7001 & 1.602 & 2.487 & 2.529 \\
& 0.034 & 0.071 & 0.101 & 0.142 \\
Circlers $(n=8)$ & $1.941^{*}$ & $1.854^{*}$ & 2.996 & 2.975 \\
& 0.147 & 0.099 & 0.301 & 0.260 \\
NAS & & & & \\
Controls $(n=7)$ & 3.866 & 3.995 & 3.375 & 3.488 \\
& 0.211 & 0.486 & 0.127 & 0.299 \\
Circlers $(n=7)$ & 4.320 & 3.820 & 3.765 & 3.750 \\
& 0.613 & 0.248 & 0.337 & 0.129
\end{tabular}

Values are expressed as mean $\mu \mathrm{g} / \mathrm{gm}$ protein $( \pm$ SEM) in tissues ipsilateral and contralateral to the direction of turning. Rats were trained to circle using a CRF schedule, as described in Materials and Methods (Experiment 1).

* Differs from appropriate control value, $p<0.05$.

tion: $F(1,6)=16.83, p<0.01$. Pairwise comparisons $(\alpha=0.05)$ indicated that relative to control values, HVA levels were bilaterally increased in rats trained to circle. The concentration of HVA in the contralateral NAS of circlers was, however, lower than within the ipsilateral NAS (see Table 3). While the bilateral enhancement of DA metabolism in rats trained to circle was further reflected in the HVA:DA ratios: $F(1,6)=20.99, p<$ 0.01 , the ratios did not indicate asymmetrical levels of DA activity (Fig. 3). Together, these results suggest that DA activity in the NAS is increased by conditioned circling. As in the striatum, changes in DA activity were not, however, restricted to the contralateral structure. While it is evident that DA function was bilaterally enhanced relative to noncircling control subjects, it should be noted that the level of activity of DA neurons contralateral to the direction of turning appears to be different from that of ipsilateral DA neurons. The lower concentrations of DA and HVA in the contralateral NAS as compared to those in the ipsilateral NAS are suggestive of an asymmetry in the extent to which dopaminergic activity is enhanced by circling. The significance of this finding is not immediately clear. If the decrease in DA reflects an increase in release and/or metabolism, then one would expect to observe a concomitant increase in metabolite concentrations. While contralateral levels of HVA were increased with respect to the control subjects, they were lower with respect to concentrations within the ipsilateral NAS. One possible explanation is that transport of the metabolites was also enhanced (see Heffner et al., 1984). If this were the case, the lower contralateral HVA concentrations may have been due to an asymmetrical increase in transport mechanisms.

Analysis of 5-HT and 5-HIAA concentrations revealed no significant differences (Table 4). However, rats trained to circle were found to have enhanced utilization of 5-HT, as estimated by the ratio of 5-HIAA to 5-HT: $F(1,6)=9.366, p<0.01$ (Fig. 3).

Olfactory tubercle. As indicated in Table 3, the concentrations of DA, DOPAC, and HVA in the OT were not influenced by performance of the conditioned circling response. In addition, there was no evidence of altered levels of activity when ratios of the metabolites to DA were analyzed (Fig. 4). Enhancement of the activity of 5-HT terminals bilaterally in rats trained to circle is suggested by increased levels of 5-HIAA: $F(1,6)=15.26$, $p<0.01$ (Table 4). This effect is also indicated when ratios of 5-HIAA to 5-HT are analyzed: $F(1,6)=25.74, p<0.01$ (see Fig. 4).

Taken together, the results of this experiment indicate that high levels of circling behavior induced and maintained by positive reinforcement can produce selective neurochemical changes in intact rats. Specifically, the turnover of DA, as estimated by HVA:DA ratios, is increased bilaterally in both the striatum and the NAS of rats trained to circle, relative to noncircling controls. The present results also indicate that conditioned circling, a behavior that is considered to be a lateralized response, produces some asymmetrical changes in the mesolimbic DA
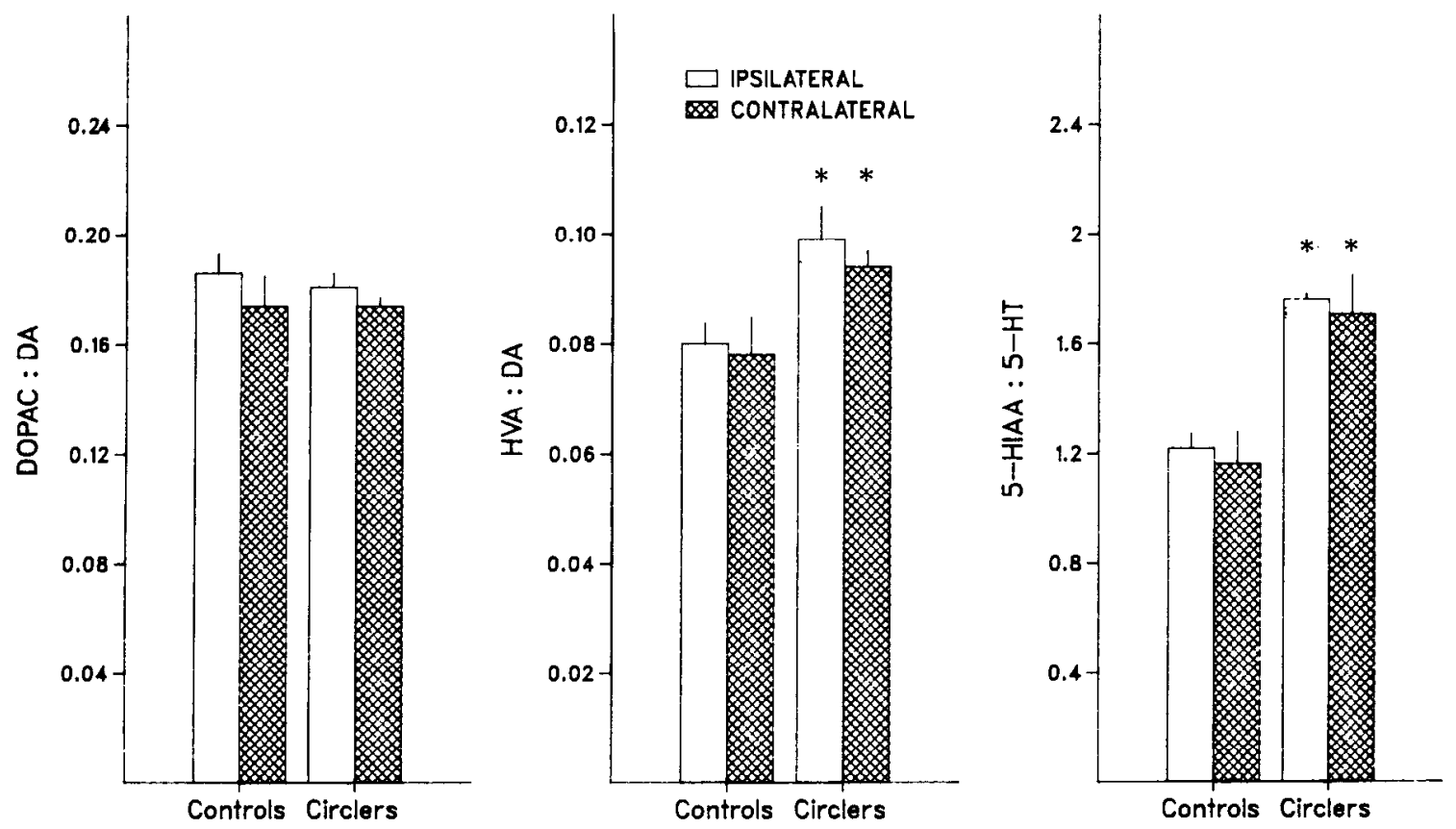

Figure 2. Ratios of DOPAC and HVA to DA and 5-HIAA to 5-HT in striatum ipsilateral and contralateral to direction of turning. Values represent the mean $( \pm$ SEM) ratios. Rats were trained to circle according to an FR-2 schedule of water reinforcement. Rats were either tested for 20 min (circlers) or were given access to water in their home cage for $20 \mathrm{~min}$ (controls) before being decapitated. Ipsilateral/contralateral designation of control values was random. *, Differs from respective control value. 
Table 3. Concentrations of DA, DOPAC, and HVA in various brain regions of circling and noncircling control rats

\begin{tabular}{|c|c|c|c|c|c|c|}
\hline & \multicolumn{2}{|c|}{ Dopamine } & \multicolumn{2}{|c|}{ DOPAC } & \multicolumn{2}{|l|}{ HVA } \\
\hline & Ipsi & Contra & Ipsi & Contra & Ipsi & Contra \\
\hline \multicolumn{7}{|l|}{ Striatum } \\
\hline Controls $(n=3)$ & $\begin{array}{r}129.33 \\
6.74\end{array}$ & $\begin{array}{r}131.00 \\
7.37\end{array}$ & $\begin{array}{r}24.03 \\
1.25\end{array}$ & $\begin{array}{r}22.80 \\
2.10\end{array}$ & $\begin{array}{r}10.33 \\
0.55\end{array}$ & $\begin{array}{r}10.18 \\
0.62\end{array}$ \\
\hline Circlers $(n=4)$ & $\begin{array}{r}124.50 \\
3.71\end{array}$ & $\begin{array}{r}117.75 \\
1.93\end{array}$ & $\begin{array}{r}22.55 \\
0.64\end{array}$ & $\begin{array}{r}20.53 \\
0.59\end{array}$ & $\begin{array}{r}12.40 \\
0.95\end{array}$ & $\begin{array}{r}11.08 \\
0.35\end{array}$ \\
\hline \multicolumn{7}{|l|}{ NAS } \\
\hline Controls $(n=3)$ & $\begin{array}{r}102.83 \\
6.58\end{array}$ & $\begin{array}{r}105.73 \\
4.84\end{array}$ & $\begin{array}{r}25.53 \\
0.81\end{array}$ & $\begin{array}{r}26.80 \\
0.52\end{array}$ & $\begin{array}{l}8.24 \\
0.23\end{array}$ & $\begin{array}{l}9.00 \\
0.60\end{array}$ \\
\hline Circlers $(n=5)$ & $\begin{array}{r}99.34 \\
2.95\end{array}$ & $\begin{array}{c}94.08^{* *} \\
2.21\end{array}$ & $\begin{array}{r}27.48 \\
0.58\end{array}$ & $\begin{array}{r}25.54 \\
0.52\end{array}$ & $\begin{array}{c}11.80^{*} \\
0.38\end{array}$ & $\begin{array}{r}10.70^{*} \\
0.53^{* *}\end{array}$ \\
\hline \multicolumn{7}{|l|}{ OT } \\
\hline Controls $(n=3)$ & $\begin{array}{r}64.87 \\
3.36\end{array}$ & $\begin{array}{r}71.17 \\
4.35\end{array}$ & $\begin{array}{r}14.60 \\
1.12\end{array}$ & $\begin{array}{r}15.43 \\
1.19\end{array}$ & $\begin{array}{l}3.99 \\
0.78\end{array}$ & $\begin{array}{l}4.46 \\
0.49\end{array}$ \\
\hline Circlers $(n=5)$ & $\begin{array}{r}64.48 \\
4.39\end{array}$ & $\begin{array}{r}61.02 \\
3.11\end{array}$ & $\begin{array}{r}15.48 \\
1.70\end{array}$ & $\begin{array}{r}13.78 \\
0.75\end{array}$ & $\begin{array}{l}5.20 \\
0.36\end{array}$ & $\begin{array}{l}4.83 \\
0.40\end{array}$ \\
\hline
\end{tabular}

Values are expressed as mean $\mu \mathrm{g} / \mathrm{gm}$ protein $( \pm \mathrm{SEM})$ in tissues ipsilateral and contralateral to the direction of turning. Rats were trained to circle using an FR-2 schedule, as described in Materials and Methods (Experiment 2).

* Differs from appropriate control value, $p<0.05$.

** Differs from own ipsilateral value, $p<0.05$.

system but not in the nigrostriatal DA projection. That is, some of the data suggest that the activity within the NAS contralateral to the direction of circling may be augmented to a greater extent than it is in the ipsilateral NAS. The failure to obtain evidence of asymmetrical DA activity within the striatum is surprising. First, this result is contrary to the findings of Yamamoto and Freed (1982, 1984). Moreover, on the basis of experiments assessing the neural basis of circling induced by unilateral lesions of the substantia nigra or striatum, it has generally been accepted that circling reflects an asymmetry in nigrostriatal DA function. In contrast, the present results clearly indicate that the turnover of DA within the striatum, as estimated by HVA:DA ratios, is increased bilaterally in circling rats.

In the present experiment, HVA appeared to be more sensitive to the effects of circling than DOPAC, the other major metabolite of DA. This finding may reflect an enhancement in the rate of conversion of DOPAC to HVA. Also, further experiments are required to determine if the rate of elimination of DA metabolites is augmented in rats trained to circle for water and, moreover, if such a change is asymmetrical.

The turnover of 5-HT, as estimated from metabolite to neurotransmitter ratios, was also found to be increased bilaterally within the striatum, NAS, and OT of rats trained to circle. These findings are also contrary to results reported by Yamamoto and Freed (1984). These authors reported that 5-HT turnover was selectively increased in the striatum and NAS contralateral to the direction of circling (the OT was not examined). The symmetrical changes in 5-HT metabolism obtained in the present experiment suggest that 5-HT may play a role in more general behavioral processes (e.g., arousal, attention, etc.) that are involved in the performance of the conditioned circling response (see, for example, Rosecrans, 1970).

\section{Discussion}

The present results provide further evidence that DA is involved in the performance of a circling response. While some evidence of asymmetrical changes in DA activity was obtained in animals trained to circle using an intermittent schedule of reinforcement, the results are quantitatively and qualitatively different from those reported by Yamamoto and Freed (1982, 1984). First, asymmetrical neurochemical changes were evident only within the NAS in the present experiments. Specifically, DA concentrations tended to be lower in the NAS contralateral to the animal's direction of turning. In contrast, the former reports indicated that circling resulted in increased levels of DA in the NAS and striatum contralateral to direction of circling. In addition, the effects of circling on DA metabolite concentrations differed substantially from Yamamoto and Freed's findings (1982). While there were no effects on the absolute levels of

Table 4. Concentrations of 5-IIT and 5-HIAA in various brain regions of circling and noncircling control rats

\begin{tabular}{|c|c|c|c|c|}
\hline & \multicolumn{2}{|l|}{$5-\mathrm{HT}$} & \multicolumn{2}{|c|}{ 5-HIAA } \\
\hline & Ipsi & Contra & Ipsi & Contra \\
\hline \multicolumn{5}{|l|}{ Striatum } \\
\hline \multirow[t]{2}{*}{ Controls $(n=3)$} & 2.007 & 2.180 & 2.446 & 2.540 \\
\hline & 0.190 & 0.170 & 0.270 & 0.340 \\
\hline \multirow[t]{2}{*}{ Circlers $(n=5)$} & 1.619 & 1.628 & 2.863 & 2.771 \\
\hline & 0.204 & 0.072 & 0.374 & 0.217 \\
\hline \multicolumn{5}{|l|}{ NAS } \\
\hline \multirow[t]{2}{*}{ Controls $(n=3)$} & 2.065 & 2.312 & 2.382 & 2.422 \\
\hline & 0.378 & 0.190 & 0.262 & 0.021 \\
\hline \multirow[t]{2}{*}{ Circlers $(n=5)$} & 2.330 & 2.058 & 3.112 & 2.971 \\
\hline & 0.242 & 0.293 & 0.179 & 0.311 \\
\hline \multicolumn{5}{|l|}{ OT } \\
\hline \multirow[t]{2}{*}{ Controls $(n=3)$} & 5.325 & 6.068 & 1.924 & 2.248 \\
\hline & 1.170 & 0.236 & 0.368 & 0.288 \\
\hline \multirow[t]{2}{*}{ Circlers $(n=5)$} & 6.666 & 6.399 & $3.233^{*}$ & $3.044^{*}$ \\
\hline & 0.363 & 0.304 & 0.231 & 0.035 \\
\hline
\end{tabular}

Values are expressed as mean $\mu \mathrm{g} / \mathrm{gm}$ protein $( \pm \mathrm{SEM})$ in tissues ipsilateral and contralateral to the direction of turning. Rats were trained to circle using an FR-2 schedule, as described in Materials and Methods (Experiment 2).

* Differs from appropriate control value, $p<0.05$. 

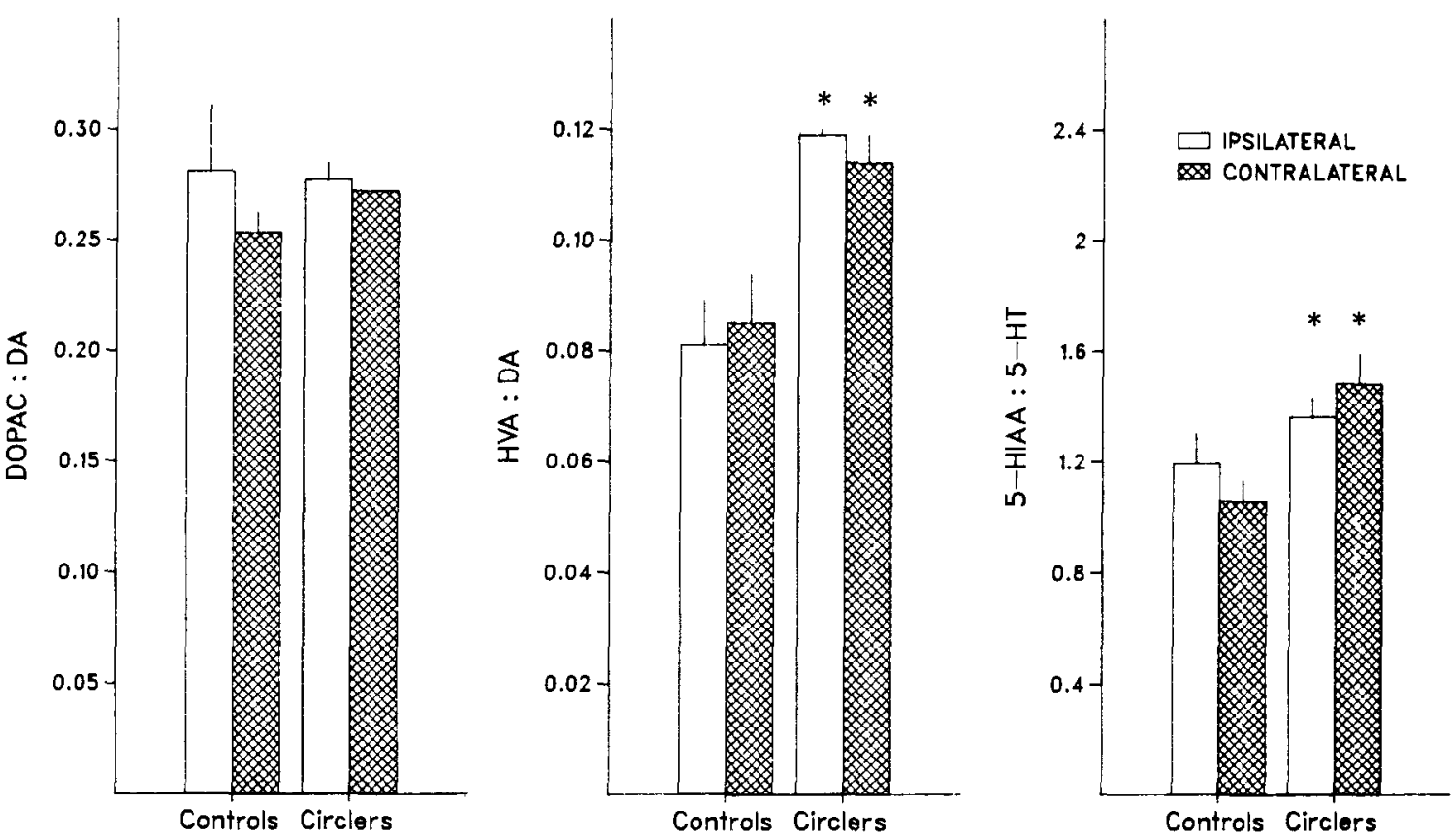

Figure 3. Ratios of DOPAC and HVA to DA and 5-HIAA to 5-HT in NAS ipsilateral and contralateral to direction of circling. Values represent the mean $\left( \pm\right.$ SEM) ratios. See Figure 2 for procedural details. ${ }^{*}$, Differs from respective control value.

either DOPAC or HVA in the striatum and OT, HVA in the NAS was elevated bilaterally. The extent of the increase was, however, less in the NAS contralateral to the direction of turning. Evidence for enhanced utilization was also obtained when HVA:DA ratios were examined. It should be noted that, when the ratios were analyzed, there was no indication of asymmetrical levels of activity within the NAS. In contrast, Yamamoto and Freed (1982) reported increases in DOPAC concentrations in both the contralateral striatum and NAS (HVA was not measured).

One possible explanation for these different results concerns the relative levels of responding obtained at the time when animals were decapitated. That is, while Yamamoto and Freed reported maximal neurochemical changes following $20 \mathrm{~min}$ of circling, the neurochemical correlates of circling may be critically dependent on the profile of responding. In both Experiments 1 and 2, rates of responding had declined by the end of the testing session. Thus, it is possible that different neurochemical effects would have been obtained had animals been killed at the time when maximal response levels occurred.

The differences between the present results and those reported by Yamamoto and Freed (1982) may also be related to different housing procedures. In the present experiments, all subjects were housed individually. However, Yamamoto and Freed typically maintained their animals in groups of three or four (C. R. Freed, personal communication). Given the evidence that environmental factors, such as housing conditions, can influence neurochemical variables and behavior (Segal et al., 1973; Thoa et al., 1977), it remains possible that this procedural difference underlies these discrepancies.

Several manipulations could be undertaken in an attempt to increase the magnitude of the present effects and to further clarify the specific neural processes involved in circling. The findings that asymmetries in striatal DA function are highly correlated with positional/directional biases (Glick et al., 1977) and that the magnitude and direction of drug-induced circling depends on whether the dominant or nondominant nigrostriatal projection is lesioned (Glick et al., 1977; Robinson and Becker, 1983) suggest that prescreening of rats to determine the preferred direction of turning may serve to magnify the degree of behaviorally induced, lateralized neurochemical changes. If an animal is trained to turn against its preferred direction, then the activity in the contralateral striatum and NAS may be increased just to the point where absolute concentrations of DA and metabolites are in balance, or just slightly higher on the contralateral side. However, were the animal required to turn in its preferred direction, the detected magnitude of the asymmetry might be larger-even though the absolute extent of neurochemical change would be constant (see Robinson and Becker, 1983). In the present series of experiments, the direction of circling was determined randomly for each animal since this was the procedure employed by Yamamoto and Freed $(1982,1984)$. It was, however, observed that some animals acquired the response more readily than others. It is possible that some subjects were being trained to turn against their inherent positional biases, and thus, the detectability of circling-induced neurochemical changes may have been limited. It should be noted that prescreening of animals would not necessarily entail the use of drugs normally used to test for directional preferences (e.g., amphetamine), as it has recently been reported that position biases detected in a T-maze apparatus are correlated with directional biases observed in drug-induced rotation tests (Dunnett et al., 1981; Glick et al., 1977).

The detection of behaviorally induced neurochemical asymmetries might be facilitated if finer-grained dissections were conducted, as the striatum is not a homogenous structure in terms of its anatomical connections, neurochemical content, or behavioral function. For example, it has been reported that the bchavioral cffects of microinjections of DA dircctly into the striatum depend on the locus of the injection site. Infusions into the dorsal striatum result in contraversive circling, while injections into the ventral striatum are either ineffective or induce slight tendencies for contraversive circling. Injections of vehicle solutions into the ventral striatum, however, result in low levels of ipsiversive circling (Joyce et al., 1982). Thus, it has been suggested that the dorsal and ventral aspects of the striatum are potentially antagonistic in their functions. If this is the case, then one might expect to find increases in the dorsal striatum 

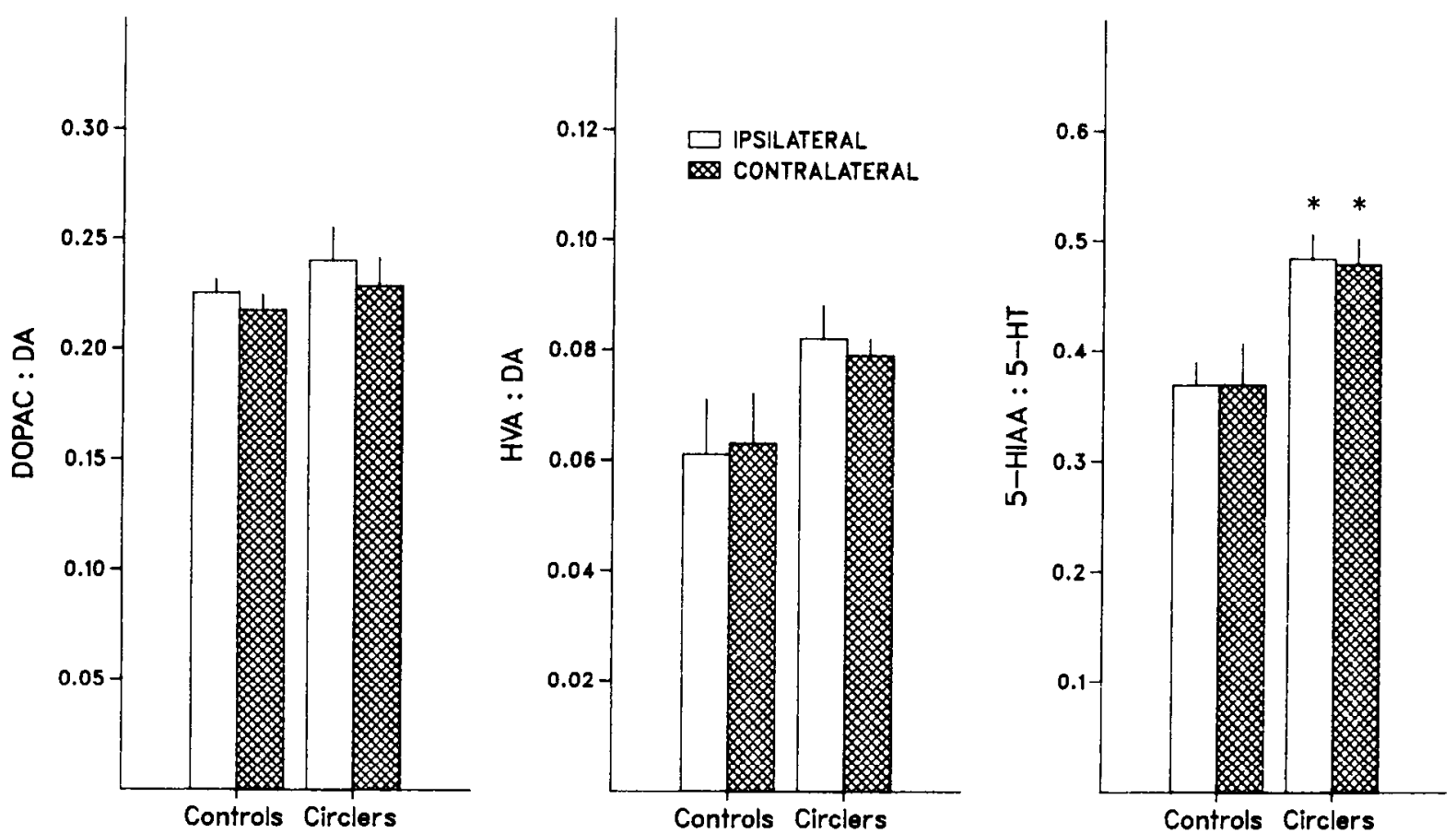

Figure 4. Ratios of DOPAC and HVA to DA and 5-HIAA to 5-HT in OT ipsilateral and contralateral to direction of turning. Values represent the mean $( \pm$ SEM) ratios. See Figure 2 for procedural details. *, Differs from respective control value.

but not in the ventral striatum. Consequently, the magnitude of the neurochemical effects may have been masked in the present series of experiments, as the ventral striatum was included in the sample of striatal tissue. Thus, more discrete dissections of the striatum may enhance neurochemical asymmetries.

Further characterization of the neural processes involved in performance of the rotational response is required. For example, it would be of interest to determine whether DA receptor modification occurs to compensate for the altered release of neurotransmitters. The response topography of the well-established circling response observed in the present experiments (i.e., tight radius, head-to-tail circling) is reminiscent of the style of circling induced by the administration of apomorphine to lesioned subjects (see Pycock, 1980). Since it has been suggested that the contraversive circling observed with apomorphine reflects DA receptor supersensitivity, the possibility that conditioned circling involves changes in receptor sensitivity should be entertained. Accordingly, it would be of interest to assess the influence of conditioned circling on DA receptors.

The consistent bilateral enhancement of 5-HT utilization, as estimated by 5 -HIAA:5-HT ratios, deserves further consideration. While it is not clear at this time what precise role ascending 5-HT projections play in circling, the present results suggest that they are involved in the response. To this end, it is interesting to note that 5 -HT activity is also augmented bilaterally in the striatum and OT of rats that exhibit circling in response to electrical stimulation of the lateral hypothalamus (Szostak et al., in press).

To conclude, the present results support previous suggestions that DA systems are involved in circling. However, the failure to observe robust asymmetrical changes in DA activity is inconsistent with the hypothesis that circling is mediated by an asymmetry in the functioning of DA systems. The lack of asymmetrical changes in $\mathrm{DA}$ activity may reflect the possibility that the conditioned circling response is not a truly lateralized behavior. While the two sides of the body are clearly not performing the same movements, it is important to appreciate that the ipsilateral side is not inactive during circling - rather, both sides are actively involved in the performance of the circling response. As such, the bilateral effects on DA activity observed in Experiment 2 may be attributable to the generally high levels of bilateral motor activity exhibited by the animals. Even if the conditioned circling response is considered to be a strongly lateralized behavior, the present results indicate that the nigrostriatal and mesolimbic DA systems are bilaterally activated by high levels of this response. This finding is open to several interpretations. It could indicate that in the awake, intact animal activation of one dopaminergic system during lateralized motor activity causes, by multisynaptic mechanisms, the other side to be activated as well. Alternatively, the bilateral activation could indicate that these systems mediate other aspects of the circling response, such as the high lcvcls of arousal and/or motivation that are present during circling. Finally, the present results raise the possibility that different neural processes underlie circling induced with different paradigms (e.g., conditioned circling and drug-induced circling). In this regard, it is interesting to note that the overall neurochemical profile associated with circling induced by intracranial electrical stimulation is substantially different from the results obtained in the present series of experiments (Szostak et al., in press).

\section{References}

Dunnett, S. B., A. Bjorklund, U. Stenevi, and S. Iversen (1981) Behavioral recovery following transplantation of substantia nigra in rats subjected to 6-OHDA lesions of the nigrostriatal pathway. I. Unilateral lesions. Brain Res. 215: 358-360.

Gershanik, O., R. Heikkila, and R. C. Duvoisin (1983) Effects of dopamine depletion on rotational behavior to dopamine agonists. Brain Res. 261: 147-161.

Glick, S. D., T. P. Jerussi, and B. Zimmerberg (1977) Behavioral and neuropharmacological correlates of nigrostriatal asymmetry in rats. In Lateralization in the Nervous System, S. Harnad, R. W. Doty, L. Goldstein, J. Jaynes, and G. Krauthamer, eds., pp. 213-249, Academic, New York.

Heffner, T. G., and L. S. Seiden (1980) Synthesis of catecholamines from $\left[{ }^{3} \mathrm{H}\right]$ tyrosine in brain during the performance of operant behavior. Brain Res. 183: 403-419. 
Heffner, T. G., D. Luttinger, J. A. Hartman, and L. S. Seiden (1981) Regional changes in brain catecholamine turn-over in the rat during performance on fixed ratio and variable interval schedules of reinforcement. Brain Res. 214: 215-218.

Heffner, T. G., G. Vosmer, and L. S. Seiden (1984) Increased transport of 3,4-dihydroxyphenylacetic acid from brain during performance of operant behavior in the rat. Brain Res. 293: 85-91.

Jakubovic, A., D. Fu, and H. C. Fibiger (in press) Factors affecting the stability and separation of biogenic amines and their metabolites: Simultaneous measurement by HPLC with electrochemical detection. J. Pharmacol. Methods.

Jerussi, T. P., and C. A. Taylor (1982) Bilateral asymmetry in striatal dopamine metabolism: Implications for pharmacotherapy of schizophrenia. Brain Res. 246: 71-75.

Joyce, J. N., and C. van Hartesveldt (1984) Rotation and postural deviation elicited by microinjections of dopamine into medial and lateral regions of dorsal striatum. Pharmacol. Biochem. Behav. 21: 979-981.

Joyce, J. N., R. E. Davis, and C. van Hartesveldt (1982) Behavioral effects of unilateral dopamine injections into dorsal or ventral striatum. Eur. J. Pharmacol. 72: 1-10.

Kelly, P. H., and K. E. Moore (1977) Mesolimbic dopamine neurons: Effects of 6-hydroxydopamine-induced destruction and receptor blockade on drug-induced rotation in rats. Psychopharmacology 55: 35-41.

Lavielle, S., J. Tassin, A. Thierry, G. Blanc, D. Herve, C. Barthelemy, and J. Glowinski (1978) Blockade by benzodiazepines of the selective high increase in dopamine turnover induced by stress in mesocortical dopaminergic neurons of the rat. Brain Res. 168: 585-594.

Pycock, C. J. (1980) Turning behavior in animals. Neuroscience 5: 461-514.

Pycock, C. J., and C. D. Marsden (1978) The rotating rodent: A two component system? Eur. J. Pharmacol. 47: 167-175.

Robinson, T. E., and J. B. Becker (1983) The rotational behavior model: Asymmetry in the effects of unilateral 5-OHDA lesions of the substantia nigra. Brain Res. 264: 127-131.

Rosecrans, J. A. (1970) Differences in brain area 5-hydroxytryptamine turnover and rearing behavior in rats and mice of both sexes. Eur. J. Pharmacol. 9: 379-382.

Rosen, G. D., S. Finkelstein, A. L. Stoll, D. A. Yutzey, and V. H. Denenberg (1984) Neurochemical asymmetries in the albino rat's cortex, striatum and nucleus accumbens. Life Sci. 34: 1143-1149.

Segal, D. S., S. Knapp, R. T. Kuczenski, and A. J. Mandell (1973) The effects of environmental isolation on behavior and regional rat brain tyrosine hydroxylase and tryptophan hydroxylase activity. Behav. Biol. 8: 47-53.

Szostak, C., A. Jakubovic, A. G. Phillips, and H. C. Fibiger (in press) Dopaminergic and serotonergic correlates of stimulation-induced circling. Brain Res.

Thoa, N. B., Y. Tizabi, and D. M. Jacobowitz (1977) The effect of isolation on catecholamine concentration and turnover in discrete areas of the brain. Brain Res. 131: 259-269.

Ungerstedt, U. (1971a) Striatal dopamine release after amphetamine or nerve degeneration revealed by rotational behavior. Acta Physiol. Scand. (Suppl.) 367: 49-68.

Ungerstedt, U. (1971b) Postsynaptic supersensitivity after 6-hydroxydopamine induced degeneration of the nigrostriatal dopamine system. Acta Physiol. Scand. (Suppl.) 367: 69-93.

Wolfson, L. I., and L. L. Brown (1983) Intrastriatal injection of $\left[{ }^{3} \mathrm{H}\right]$ dopamine through a chronic cannula to produce rotation: Distribution and concentration of the tracer in specific brain regions. Brain Res. 261: 205-212.

Yamamoto, B. K., and C. R. Freed (1982) The trained circling rat: A model for inducing unilateral caudate dopamine metabolism. Nature 298: 467-468.

Yamamoto, B. K., and C. R. Freed (1984) Asymmetric dopamine and serotonin metabolism in nigrostriatal and limbic structures of the trained circling rat. Brain Res. 297: 115-119. 